\title{
Does robotic radical cystectomy impede oncological outcome in bladder cancer patients?
}

\author{
Günter Niegisch \\ Department of Urology, Medical Faculty, Heinrich-Heine-University, Düsseldorf, Germany \\ Correspondence to: Günter Niegisch. Department of Urology, Medical Faculty, Heinrich Heine University Duesseldorf, Moorenstr 5, 40225 \\ Duesseldorf, Germany. Email: guenter.niegisch@hhu.de. \\ Provenance: This is an invited Editorial commissioned by Section Editor Xiao Li (Department of Urology, Jiangsu Cancer Hospital \& Jiangsu \\ Institute of Cancer Research \& Nanjing Medical University Affiliated Cancer Hospital, Nanjing, China). \\ Comment on: Bochner BH, Dalbagni G, Marzouk KH, et al. Randomized Trial Comparing Open Radical Cystectomy and Robot-assisted \\ Laparoscopic Radical Cystectomy: Oncologic Outcomes. Eur Urol 2018;74:465-71.
}

Submitted Aug 06, 2018. Accepted for publication Aug 13, 2018.

doi: $10.21037 /$ tau.2018.08.16

View this article at: http://dx.doi.org/10.21037/tau.2018.08.16

In recent years, new surgical procedures have expanded the urological treatment spectrum. In addition to a number of new developments in urological endoscopy, such as fluorescence-assisted transurethral resection of bladder tumours or improved laser systems, these include minimally invasive surgical techniques in "major" uro-oncologic surgery. In particular, the use of robot-assisted methods is becoming more important, as they play an important role in patient awareness, not least because of offensive marketing.

If new procedures are implemented in medical routine, the quality of patient care must not decrease. It must be shown that the new methods are also at least equivalent, but reasonably superior, to established methods even outside of clinical studies.

In this context, Bochner and his colleagues set up a prospective randomized trial comparing traditional open radical cystectomy (ORC) to robot-assisted radical cystectomy (RARC). The primary outcome of this trial was overall $90-\mathrm{d}$ grade $2-5$ complications defined by a modified Clavien system (1). Results of this trial which enrolled 118 patients (60 RARC, 58 ORC) focussing on the primary outcome as well as on a number of further relevant perioperative outcomes have been published in 2014 and $2015(2,3)$. In summary, neither 90-d grade 2-5 complication rate nor other relevant perioperative parameters (margin status, lymph node yield, quality of life, differed comparing ORC and RARC. Though, the authors observed a lower transfusion rate and less wound complications in RARC patients while OR time was longer and costs were higher for RARC patients. While this level I evidence as well as the results from a number of other prospective and retrospective analysis (4-9) clearly showed that perioperative patients' safety is not impeded by RARC, there is still an ongoing debate on oncological outcomes of RARC as compared to ORC. In particular, this debate has been fuelled by recent controversial reports regarding differences in recurrence patterns comparing ORC to RARC and a higher rate of early recurrence comparing ORC to laparoscopic radical cystectomy (10-12). Therefore, the oncological follow-up data reported by Bochner et al. in this recent post-hoc analysis of their prospective randomized trial is of major importance.

\section{Summary of the study results}

A total of 118 patients have been included in this analysis (60 RARC, 58 ORC). Baseline characteristics of both groups were balanced, though more male patients were included in the RARC group and pathological pT0 stage was more frequent in the RARC group (probably more complete responses following neoadjuvant chemotherapy). The authors did not observe any relevant difference in oncological outcomes comparing ORC and RARC patients after a median follow-up time of 4.9 years. Recurrencefree survival as well as bladder-cancer-specific and all-cause mortality were similar. Regarding recurrence pattern, ORC patients trended to develop more distant recurrences, while local/regional recurrences seemed to be more frequent in 
RARC patients. However, acknowledging giving limitations of their secondary analysis (the trial was not powered to detect differences regarding oncological outcomes or differences in recurrence pattern) the authors draw conclusions carefully and state that "The secondary outcomes from our randomized trial did not definitively demonstrate differences in cancer outcomes in patients treated with ORC or $R A R C$ " and "The wide CIs (confidence intervals) around the difference in recurrence risk preclude us from making conclusions regarding oncologic equivalence of the surgical modalities."

So, given his careful conclusions, do we need still to fear that, in the long run of oncological outcome, we may harm our patients by offering them a modern surgical approach to radical cystectomy? I do not think so.

\section{Oncological survival data}

While indeed the data presented by Bochner et al. may be impeded by given limitations as study design and a selected patient population (e.g., few patients with extravesical disease, a rather high rate of patients treated with neoadjuvant chemotherapy), their results are supported by a number of further studies focussing on oncological outcomes in RARC patients. For example, in the RAZOR (RARC versus ORC in patients with bladder cancer) trial, no differences in 2-year progression-free survival rate comparing ORC and RARC patients (RARC $72.3 \%$, ORC $71.6 \%)$ was observed indicating non-inferiority of the robotic approach. In addition, a number of retrospective analysis support these findings as well (13-15). In fact, these results are not unexpected, as RARC has already been shown to be safe regarding perioperative predictors of oncological outcomes as lymph node yield and margin status. In my opinion, integrating radical cystectomy in a multimodal approach with consequent perioperative systemic therapy (neoadjuvant and adjuvant chemotherapy) is more relevant for the patient outcome than the surgical approach. Accordingly, research efforts now should focus on this field (immunotherapy, radiation therapy, small molecules, combinatorial treatments).

\section{Recurrence pattern}

The second important issue is whether the surgical approach may trigger the pattern of recurrence in relapsing patients. Indeed, Bochner et al. report that in RARC patients, local/regional relapse was observed more frequently while distant metastases were more frequent in ORC patients. There are other studies, which report differences in metastatic pattern or unexpected metastatic pattern triggered by the surgical as well. For example, Albisinni et al. report on unexpected metastatic sites (scapula, corpora cavernosa and axillary lymph nodes) in recurrent patients following laparoscopic radical cystectomy and Nguyen et al. observed extrapelvic lymphatic and peritoneal metastases more frequently in RARC patients ( $23 \%$ vs. $15 \%, 21 \%$ vs. $8 \%)(10,11)$. However, in my opinion, these differences are more likely to be triggered rather by tumor biology than by the surgical approach as reported in a follow-up study in a follow-up study on 310 RARC patients by Nguyen et al. as well (16). Further, from the oncologist's perspective, in the end it does not really matter for the patient where but that he relapses following curative intended radical cystectomy. Nevertheless, from a scientific perspective these findings deserve a closer look not only including surgical but also biological factors.

\section{Conclusions}

In summary, the study of Bochner et al. underlines that we may offer RARC to our patients not only without impeding perioperative but also oncological safety. Neither progression-free nor cancer-specific survival is impaired by RARC and findings regarding differences in pattern of relapse between ORC and RARC are, in my opinion, only of minor relevance for the patient.

Nevertheless, prospective scientific evaluation of RARC following strict documentation of perioperative and followup date is further of major importance and therefore mandatory, as there still remain unanswered question, e.g., long-term functional outcomes depending on the technique of diversion or long-term complications.

\section{Acknowledgements}

None.

\section{Footnote}

Conflicts of Interest: The author has no conflicts of interest to declare.

\section{References}

1. Shabsigh A, Korets R, Vora KC, et al. Defining early 
morbidity of radical cystectomy for patients with bladder cancer using a standardized reporting methodology. Eur Urol 2009;55:164-74.

2. Bochner BH, Dalbagni G, Sjoberg DD, et al. Comparing Open Radical Cystectomy and Robot-assisted Laparoscopic Radical Cystectomy: A Randomized Clinical Trial. Eur Urol 2015;67:1042-50.

3. Bochner BH, Sjoberg DD, Laudone VP, et al. A randomized trial of robot-assisted laparoscopic radical cystectomy. N Engl J Med 2014;371:389-90.

4. Niegisch G, Albers P, Rabenalt R. Perioperative complications and oncological safety of robot-assisted (RARC) vs. open radical cystectomy (ORC). Urol Oncol 2014;32:966-74.

5. Khan MS, Gan C, Ahmed K, et al. A Single-centre Early Phase Randomised Controlled Three-arm Trial of Open, Robotic, and Laparoscopic Radical Cystectomy (CORAL). Eur Urol 2016;69:613-21.

6. Nix J, Smith A, Kurpad R, et al. Prospective randomized controlled trial of robotic versus open radical cystectomy for bladder cancer: perioperative and pathologic results. Eur Urol 2010;57:196-201.

7. Kader AK, Richards KA, Krane LS, et al. Robot-assisted laparoscopic vs open radical cystectomy: comparison of complications and perioperative oncological outcomes in 200 patients. BJU Int 2013;112:E290-4.

8. $\mathrm{Ng} \mathrm{CK}, \mathrm{Kauffman}$ EC, Lee MM, et al. A comparison of postoperative complications in open versus robotic cystectomy. Eur Urol 2010;57:274-81.

9. Parekh DJ, Messer J, Fitzgerald J, et al. Perioperative outcomes and oncologic efficacy from a pilot prospective randomized clinical trial of open versus robotic assisted radical cystectomy. J Urol 2013;189:474-9.

Cite this article as: Niegisch G. Does robotic radical cystectomy impede oncological outcome in bladder cancer patients? Transl Androl Urol 2018;7(Suppl 6):S744-S746. doi: 10.21037/tau.2018.08.16
10. Nguyen DP, Al Hussein Al Awamlh B, Wu X, et al. Recurrence patterns after open and robot-assisted radical cystectomy for bladder cancer. Eur Urol 2015;68:399-405.

11. Albisinni S, Fossion L, Oderda M, et al. Critical Analysis of Early Recurrence after Laparoscopic Radical Cystectomy in a Large Cohort by the ESUT. J Urol 2016;195:1710-7.

12. Hussein AA, Saar M, May PR, et al. Early Oncologic Failure after Robot-assisted Radical Cystectomy: Results from the International Robotic Cystectomy Consortium. J Urol 2017;197:1427-36.

13. Niegisch G, Nini A, Michalski R, et al. Comparison of 2-Year Oncological Outcome and Early Recurrence Patterns in Patients with Urothelial Bladder Carcinoma Treated with Open or Robot-Assisted Radical Cystectomy with an Extracorporeal Urinary Diversion. Urol Int 2018;101:224-31.

14. Kim TH, Sung HH, Jeon HG, et al. Oncological Outcomes in Patients Treated with Radical Cystectomy for Bladder Cancer: Comparison Between Open, Laparoscopic, and Robot-Assisted Approaches. J Endourol 2016;30:783-91.

15. Simone G, Tuderti G, Misuraca L, et al. Perioperative and mid-term oncologic outcomes of robotic assisted radical cystectomy with totally intracorporeal neobladder: Results of a propensity score matched comparison with open cohort from a single-centre series. Eur J Surg Oncol 2018;44:1432-8.

16. Nguyen DP, Al Hussein Al Awamlh B, O'Malley P, et al. Factors Impacting the Occurrence of Local, Distant and Atypical Recurrences after Robot-Assisted Radical Cystectomy: A Detailed Analysis of 310 Patients. J Urol 2016;196:1390-6. 